\title{
Finite-Time Stability Analysis for a Class of Continuous Switched Descriptor Systems
}

\author{
Pan Tinglong, Yang Kun, Shen Yanxia, Gao Zairui, and Ji Zhicheng \\ Key Laboratory of Advanced Process Control for Light Industry, Jiangnan University, Ministry of Education, Wuxi 214122, China \\ Correspondence should be addressed to Pan Tinglong; tlpan@jiangnan.edu.cn
}

Received 9 September 2013; Revised 3 November 2013; Accepted 26 December 2013; Published 8 January 2014

Academic Editor: Shihua Li

Copyright (C) 2014 Pan Tinglong et al. This is an open access article distributed under the Creative Commons Attribution License, which permits unrestricted use, distribution, and reproduction in any medium, provided the original work is properly cited.

\begin{abstract}
Finite-time stability has more practical application values than the classical Lyapunov asymptotic stability over a fixed finite-time interval. The problems of finite-time stability and finite-time boundedness for a class of continuous switched descriptor systems are considered in this paper. Based on the average dwell time approach and the multiple Lyapunov functions technique, the concepts of finite-time stability and boundedness are extended to continuous switched descriptor systems. In addition, sufficient conditions for the existence of state feedback controllers in terms of linear matrix inequalities (LMIs) are obtained with arbitrary switching rules, which guarantee that the switched descriptor system is finite-time stable and finite-time bounded, respectively. Finally, two numerical examples are presented to illustrate the reasonableness and effectiveness of the proposed results.
\end{abstract}

\section{Introduction}

Switched systems are a special class of hybrid systems, which consist of a collection of continuous or discrete-time subsystems together with a switching rule that orchestrates switching between these subsystems to achieve the control objectives [1]. Descriptor systems are also referred to as singular systems, implicit systems, or differential-algebraic systems, which are also a natural representation of dynamic systems and can describe physical systems better than the normal linear systems. Descriptor systems have been widely applied in many practical systems such as networks, power systems, electrical circuits, economics mathematical modeling, and many other fields $[2,3]$. In actual control systems, switching phenomenon of descriptor systems is ubiquitous. Nevertheless, because of the switching between multiple descriptor subsystems and the algebraic constraints in descriptor model, it is inevitably difficult to analyze and synthesize for switched descriptor systems.

Up to now, much attention has been mainly focused on system stability and reliability $[2-5], H_{\infty}$ control [6-8], costguaranteed control [9-11], system controllability, and reachability $[12,13]$ for switched descriptor systems. Generally, most of existing results related to stability and performance criteria of switched descriptor systems are based on the classical Lyapunov asymptotic stability, which is defined as an infinite time interval. However, in many actual systems, such as network control systems, the practical system state does not exceed some bound during some time interval and we need to avoid saturations and the excitation of nonlinear dynamics. In this case, the asymptotic stability is not enough for practical applications, because the system could be Lyapunov asymptotically stable but it possesses undesirable transient performances. Then the concept of finite-time stability was firstly put forward in [14], which concerns the boundedness of the system state over a fixed finite-time interval. To a certain degree, the development of the finite-time stability theory is parallel with the development of Lyapunov asymptotic stability.

In recent years, the abundant studies on finite-time stability of switched systems [15-20] and descriptor systems [2124] have been obtained. Lin et al. [15] gave some results on finite-time boundedness and finite-time weighted $L_{2}$-gain for a class of switched delay systems with time-varying exogenous disturbances. Sufficient conditions which ensure that the switched system with time-time is finite-time bounded and has finite-time weighted $L_{2}$-gain were proposed based on the average dwell time approach and the multiple Lyapunov 
functions technique. In [23], the issue of robust finite-time stabilization of descriptor stochastic systems with timevarying norm-bounded disturbance and parametric uncertainties via static output feedback was discussed. Suppose that the state vector is not available for feedback; a static output feedback controller in terms of restricted LMIs was provided to guarantee the underlying closed-loop descriptor stochastic system finite-time stabilization with a prescribed $H_{\infty} r$ disturbance attenuation over the given finite-time interval. Meanwhile, an illustrative example was employed to verify the efficiency of the proposed method. In view of the importance of practical application, we need to pay great attention to the research on finite-time stability for switched descriptor systems compared with Lyapunov asymptotic stability. Few works that deal with the finite-time stability for this type of systems have been reported. Based on the different multiple Lyapunov functions, the papers $[25,26]$ focused on the discrete-time switched descriptor systems and switched descriptor systems with time-varying delay, respectively.

The paper is organized as follows. Firstly, the concepts of finite-time stability and finite-time boundedness for normal systems are expanded to continuous switched descriptor systems. Then, based on the state transfer matrix method, the sufficient and necessary condition of finite-time stability for this kind of system is given. Moreover, we tackle the problems of state feedback finite-time stabilization and finite-time boundedness; the sufficient conditions for the existence of controllers are obtained with arbitrary switching rules, which guarantee that the closed-loop systems are finite-time stable and finite-time bounded, respectively. Detailed proofs are presented by using the multiple Lyapunov functions and the average dwell-time approach. Finally, two examples are presented to show the validity of the developed methodology. Our research results are totally different from those previous results and important supplements for stability study for switched descriptor systems.

\section{Problem Description and Preliminaries}

Consider a class of switched descriptor system as follows:

$$
\begin{gathered}
E \dot{x}(t)=A_{\sigma(t)} x(t)+B_{\sigma(t)} u_{\sigma(t)}(t)+G_{\sigma(t)} w(t), \\
\dot{w}(t)=F_{\sigma(t)} w(t),
\end{gathered}
$$

where $x(t) \in R^{n}$ is the system state, $u_{\sigma}(t) \in R^{p}$ is the control input, and $w(t) \in R^{q}$ is the exogenous disturbance signal and satisfies the constraint $w^{T}(0) w(0) \leq d, d \geq 0$; the switching signal $\sigma(t): Z^{+} \rightarrow\{1,2, \ldots, m\}$ is a piecewise constant and right continuous function; $m$ is the number of subsystems; $\sigma(t)=i$ represents that the $i$ th subsystem is activated. $E, A_{i}$, $B_{i}, G_{i}$, and $F_{i}$ are known constant matrices with appropriate dimension, and it is assumed that rank $E=r \leq n$.

Remark 1. In view of the special structure of descriptor systems, the initial condition is given as $E x\left(t_{0}\right)=E x_{0}$.
Corresponding to the switching signal $\sigma(t)$, the switching sequence is defined as follows:

$$
\left\{x_{0} ;\left(i_{0}, t_{0}\right), \ldots,\left(i_{k}, t_{k}\right), \ldots \mid i_{k} \in\{1,2, \ldots, m\}, k=0,1, \ldots\right\},
$$

which means that the $i_{k}$ th subsystem is activated when $t \in$ $\left[t_{k}, t_{k+1}\right)$ and $x_{0}, t_{0}$ are the initial state and initial time, respectively. In addition, we can transform the descriptor matrix of different form into the singular matrix " $E$ ” of system (1) by nonsingular transformation.

Assumption 2. The initial state of system (1) discussed is the consistent initial state, and the system state does not jump at the switching moment.

Now, we give the definitions of finite-time stability and finite-time boundedness for the continuous switched descriptor systems.

Definition 3. Continuous switched descriptor system

$$
\begin{gathered}
E \dot{x}(t)=A_{\sigma(t)} x(t)+G_{\sigma(t)} w(t), \\
\dot{w}(t)=F_{\sigma(t)} w(t), \quad w^{T}(0) w(0) \leq d
\end{gathered}
$$

is said to be finite-time bounded with respect to $\left(c_{1}, c_{2}, d, R, T_{f}, \sigma\right)$, if the following formula:

$$
\begin{array}{r}
x^{T}(0) E^{T} R E x(0) \leq c_{1} \Longrightarrow x^{T}(t) E^{T} R E x(t) \leq c_{2}, \\
\forall t \in\left[0, T_{f}\right],
\end{array}
$$

holds, where $c_{2}>c_{1}>0, d \geq 0, T_{f}>0$, and $R>0$.

Definition 4. Continuous switched descriptor system

$$
E \dot{x}(t)=A_{\sigma(t)} x(t)
$$

is said to be finite-time stable with respect to $\left(c_{1}, c_{2}, R, T_{f}, \sigma\right)$, if the following formula:

$$
\begin{array}{r}
x^{T}(0) E^{T} R E x(0) \leq c_{1} \Longrightarrow x^{T}(t) E^{T} R E x(t) \leq c_{2}, \\
\forall t \in\left[0, T_{f}\right],
\end{array}
$$

holds, where $c_{2}>c_{1}>0, T_{f}>0$, and $R>0$.

Definition 5 (see [15]). For any $t_{f} \geq t_{0} \geq 0$, if $N_{\sigma(t)}\left(t_{0}, t_{f}\right) \leq$ $N_{0}+\left(t_{f}-t_{0}\right) / \tau_{a}$ holds for given $N_{0} \geq 0, \tau_{a}>0$, where $N_{\sigma(t)}\left(t_{0}, t_{f}\right)$ denotes the switching number of $\sigma(t)$ over $\left[t_{0}, t_{f}\right)$, then the constant $\tau_{a}$ is called the average dwell time and $N_{0}$ is the flutter bound. As commonly used in the previous literature, we choose $N_{0}=0$.

Remark 6. Finite-time stability for norm switched descriptor systems refers to the fact that the state of slow subsystem is less than a given upper bound. According to regularity of systems, the state of fast subsystem is also less than a given upper bound. 


\section{Main Results}

Firstly, the sufficient and necessary condition of finite-time stability for system (5) is given by applying the state transition matrix method.

Theorem 7. Given positive constants $c_{1}, c_{2}$, and $T_{f}$, the system (5) is finite-time stable with respect to $\left(c_{1}, c_{2}, R, T_{f}, \sigma\right)$, if and only if

$$
\Phi_{\sigma}^{T}(t, 0) E^{T} R E \Phi_{\sigma}(t, 0) \leq \frac{c_{2}}{c_{1}} E^{T} R E, \quad \forall t \in\left[0, T_{f}\right),
$$

where $\Phi_{\sigma}(t, 0)=\Phi_{i_{k}}\left(t, t_{k}\right) \Phi_{i_{k-1}}\left(t_{k}, t_{k-1}\right) \cdots \Phi_{i_{0}}\left(t_{1}, 0\right)$ is the state transition matrix.

Proof. The following proof can be divided into two cases.

(a) Sufficiency. Since $\Phi_{\sigma}(t, 0)$ is the state transition matrix of system (5), then

$$
x(t)=\Phi_{\sigma}(t, 0) x(0), \quad t \in\left[0, T_{f}\right),
$$

where $\Phi_{\sigma}(t, 0)=\Phi_{i_{k}}\left(t, t_{k}\right) \Phi_{i_{k-1}}\left(t_{k}, t_{k-1}\right) \cdots \Phi_{i_{0}}\left(t_{1}, 0\right)$. In view of $x^{T}(0) E^{T} R E x(0) \leq c_{1}$, one obtains

$$
x^{T}(t) E^{T} R E x(t)=x^{T}(0) \Phi_{\sigma}^{T}(t, 0) E^{T} R E \Phi_{\sigma}(t, 0) x(0) .
$$

On the other hand, from (7) and $x^{T}(0) E^{T} R E x(0) \leq c_{1}$, we have

$$
x^{T}(t) E^{T} R E x(t) \leq x^{T}(0) \frac{c_{2}}{c_{1}} E^{T} R E x(0) \leq c_{2} .
$$

Thus, the system (5) is finite-time stable according to Definition 4.

(b) Necessity. Suppose that the system (5) is finite-time stable with respect to $\left(c_{1}, c_{2}, R, T_{f}, \sigma\right)$. By using the reduction to absurdity, if there exists $\operatorname{Ex}\left(t^{*}\right) \neq 0, t^{*} \in\left[0, T_{f}\right)$, such that

$$
\begin{gathered}
x^{T}\left(t^{*}\right) \Phi_{\sigma}^{T}\left(t^{*}, 0\right) E^{T} R E \Phi_{\sigma}\left(t^{*}, 0\right) x\left(t^{*}\right) \\
\geq x^{T}\left(t^{*}\right) \frac{c_{2}}{c_{1}} E^{T} R E x\left(t^{*}\right)>0 .
\end{gathered}
$$

Let $\lambda=\sqrt{c_{1} / x^{T}\left(t^{*}\right) E^{T} R E x\left(t^{*}\right)}, x_{0}=\lambda x\left(t^{*}\right)$; then we get $x_{0}^{T} E^{T} R E x_{0}=c_{1}$. By virtue of (11), we have

$$
x_{0}^{T} \Phi_{\sigma}^{T}\left(t^{*}, 0\right) E^{T} R E \Phi_{\sigma}\left(t^{*}, 0\right) x_{0} \geq x_{0}^{T} \frac{\mathcal{C}_{2}}{c_{1}} E^{T} R E x_{0} .
$$

Meanwhile, according to (8), we can obtain

$$
\begin{aligned}
x^{T}\left(t^{*}\right) & E^{T} R E x\left(t^{*}\right) \\
& =x_{0}^{T} \Phi_{\sigma}^{T}\left(t^{*}, 0\right) E^{T} R E \Phi_{\sigma}\left(t^{*}, 0\right) x_{0} \geq c_{2} .
\end{aligned}
$$

Noticing that it is inconsistent with the hypothesis that the system (5) is finite-time stable with respect to $\left(c_{1}, c_{2}, R, T_{f}, \sigma\right)$, the proof is completed.
Remark 8. From Theorem 7 we can obtain the sufficient and necessary condition, which guarantees that the switched descriptor system (5) is finite-time stable. However, it is difficult and inconvenient to calculate state transition matrix and design controller. Thus, it is difficult to apply in the actual systems.

Theorem 9. For any $i, j \in\{1,2, \ldots, m\}$, if there exist nonsingular matrix $P_{i}$, matrices $Q_{i}>0, Z_{i}>0$, and scalars $\alpha \geq 0, \mu \geq 1$ such that

$$
P_{i} E=E^{T} P_{i}^{T} \geq 0,
$$

$$
\begin{gathered}
{\left[\begin{array}{cc}
P_{i} A_{i}+A_{i}^{T} P_{i}^{T}-\alpha P_{i} E & P_{i} G_{i} \\
G_{i}^{T} P_{i}^{T} & F_{i}^{T} Q_{i}+Q_{i} F_{i}-\alpha Q_{i}
\end{array}\right]<0,} \\
P_{i} E=E^{T} R^{1 / 2} Z_{i} R^{1 / 2} E, \\
P_{i} E \leq \mu P_{j} E, \quad Q_{i} \leq \mu Q_{j}, \\
\frac{\lambda_{1} c_{1}+\lambda_{3} d}{\lambda_{2} c_{2}}<e^{-\alpha T_{f}} .
\end{gathered}
$$

And the average dwell time of the switching signal $\sigma$ satisfies

$$
\tau_{a}>\tau_{a}^{*}=\frac{T_{f} \ln \mu}{\ln \left(\lambda_{2} c_{2}\right)-\ln \left(\lambda_{1} c_{1}+\lambda_{3} d\right)-\alpha T_{f}},
$$

where $\lambda_{1}=\max _{i=1,2, \ldots, m}\left\{\lambda_{\max }\left(Z_{i}\right)\right\}, \quad \lambda_{2}=$ $\min _{i=1,2, \ldots, m}\left\{\lambda_{\min }\left(Z_{i}\right)\right\}$, and $\lambda_{3}=\max _{i=1,2, \ldots, m}\left\{\lambda_{\max }\left(Q_{i}\right)\right\}$; then system (3) is regular, pulse-free, and finite-time bounded with respect to $\left(c_{1}, c_{2}, d, R, T_{f}, \sigma\right)$.

Proof. First, from (15) we have

$$
P_{i} A_{i}+A_{i}^{T} P_{i}^{T}-\alpha P_{i} E<0 .
$$

Considering rank $E=r$ and assuming that there exist invertible matrices $M, N$, such that $\bar{E}=\operatorname{MEN}=\operatorname{diag}\left\{I_{r}, 0\right\}$, then it follows from (14) and (20) that

$$
\begin{gathered}
\bar{P}_{i} \bar{E}=\bar{E}^{T} \bar{P}_{i}^{T} \geq 0, \\
\bar{P}_{i} \bar{A}_{i}+\bar{A}_{i}^{T} \bar{P}_{i}^{T}-\alpha \bar{P}_{i} \bar{E}<0,
\end{gathered}
$$

where $\bar{E}=M E N=\operatorname{diag}\left\{I_{r}, 0\right\}, \bar{A}_{i}=M A_{i} N, \bar{P}_{i}=N^{T} P_{i} M^{-1}$. According to (21), we can obtain $\bar{P}_{i}$ as follows:

$$
\bar{P}_{i}=\left[\begin{array}{cc}
P_{i 1} & P_{i 2} \\
0 & P_{i 3}
\end{array}\right], \quad P_{i 1}>0, \operatorname{det}\left(P_{i 3}\right) \neq 0 .
$$

Correspondingly, suppose that $\bar{A}_{i}=\left[\begin{array}{cc}A_{i 11} & A_{i 12} \\ A_{i 21} & A_{i 22}\end{array}\right]$, and it follows from (22) that

$$
\left[\begin{array}{lc}
\# & \# \\
\# & P_{i 3} A_{i 22}+A_{i 22}^{T} P_{i 3}^{T}
\end{array}\right]<0,
$$

where we do not need to know the expression of \# and it does not affect the following discussion. It follows from (24) 
that $P_{i 3} A_{i 22}+A_{i 22}^{T} P_{i 3}^{T}<0$; namely, $A_{i 22}$ is nonsingular. Then, there exists a scalar $s$ such that $\operatorname{det}\left(s \bar{E}-\bar{A}_{i}\right) \neq 0$ and, for $\forall s$, $\operatorname{deg} \operatorname{det}\left(s \bar{E}-\bar{A}_{i}\right)=\operatorname{rank} \bar{E}$ holds. Thus, the system (3) is regular and pulse-free.

In the following, we will prove that system (3) is finitetime bounded with respect to $\left(c_{1}, c_{2}, d, R, T_{f}, \sigma\right)$. Choose the multiple Lyapunov function as

$$
\begin{aligned}
V(t, x(t), w(t)) & =V_{\sigma}(t, x(t), w(t)) \\
& =x^{T}(t) P_{\sigma} E x(t)+w^{T}(t) Q_{\sigma} w(t)
\end{aligned}
$$

Then, let $V(t, x(t), w(t))=V(t)$ and $V_{\sigma}(t, x(t), w(t))=V_{\sigma}(t)$. By virtue of (14) and switched sequence (2), when $t \in$ $\left[t_{k}, t_{k+1}\right)$, taking derivative of $V(t)$ with respect to $t$ along the trajectory of the system (3) yields

$$
\begin{aligned}
\dot{V} & (t) \\
& =\dot{V}_{\sigma\left(t_{k}\right)}(t) \\
& =\left[\begin{array}{c}
x(t) \\
w(t)
\end{array}\right]^{T} \\
& \times\left[\begin{array}{cc}
P_{\sigma\left(t_{k}\right)} A_{\sigma\left(t_{k}\right)}+A_{\sigma\left(t_{k}\right)}^{T} P_{\sigma\left(t_{k}\right)}^{T} & P_{\sigma\left(t_{k}\right)} G_{\sigma\left(t_{k}\right)} \\
G_{\sigma\left(t_{k}\right)}^{T} P_{\sigma\left(t_{k}\right)}^{T} & F_{\sigma\left(t_{k}\right)}^{T} Q_{\sigma\left(t_{k}\right)}+Q_{\sigma\left(t_{k}\right)} F_{\sigma\left(t_{k}\right)}
\end{array}\right] \\
& \times\left[\begin{array}{c}
x(t) \\
w(t)
\end{array}\right] .
\end{aligned}
$$

Then, it follows from (15) that

$$
\dot{V}<\alpha V=\alpha V_{\sigma\left(t_{k}\right)}(t)
$$

Integrating both sides of (27) from $t_{k}$ to $t$ gives

$$
V(t)<e^{\alpha\left(t-t_{k}\right)} V_{\sigma\left(t_{k}\right)}\left(t_{k}\right)
$$

Then, together with (17), at the switched moment $t_{k}$ we derive

$$
V(t)<e^{\alpha\left(t-t_{k}\right)} \mu V_{\sigma\left(t_{k}^{-}\right)}\left(t_{k}^{-}\right) .
$$

For any $t \in\left[0, T_{f}\right)$, assuming that $N$ is the switched number of systems between $\left[0, T_{f}\right)$, one can obtain $N_{\sigma}(0, t) \leq N$. Considering $\mu \geq 1$ and using (17) together with (29), based on iterative method, we have

$$
V(t)<e^{\alpha t} \mu^{N_{\sigma}(0, t)} V_{\sigma(0)}(0) \leq e^{\alpha t} \mu^{N} V_{\sigma(0)}(0) .
$$

Noticing that $N \leq T_{f} / \tau_{a}$, then

$$
V(t)<e^{\alpha t} \mu^{T_{f} / \tau_{a}} V_{\sigma(0)}(0)
$$

On the other hand, it follows from (16) that

$$
\begin{aligned}
V(t) \geq & x^{T}(t) P_{\sigma} \operatorname{Ex}(t) \\
\geq & \lambda_{\min }\left(Z_{\sigma}\right) x^{T}(t) E^{T} R E x(t) \\
\geq & \lambda_{2} x^{T}(t) E^{T} R E x(t) \\
V_{\sigma(0)}(0) & \\
\quad & x^{T}(0) P_{\sigma(0)} \operatorname{Ex}(0)+w^{T}(0) Q_{\sigma(0)} w(0) \\
& \leq \lambda_{\max }\left(Z_{\sigma(0)}\right) x^{T}(0) E^{T} R E x(0)+\lambda_{\max }\left(Q_{\sigma(0)}\right) d \\
& \leq \lambda_{1} x^{T}(0) E^{T} R E x(0)+\lambda_{3} d
\end{aligned}
$$

when $x^{T}(0) E^{T} R E x(0) \leq c_{1}$; putting together (31)-(32), it can easily be verified that

$$
x^{T}(t) E^{T} R E x(t)<e^{\alpha t} \mu^{T_{f} / \tau_{a}}\left(\frac{\lambda_{1} c_{1}+\lambda_{3} d}{\lambda_{2}}\right) .
$$

According to (18), we get

$$
\ln \left(\lambda_{2} c_{2}\right)-\ln \left(\lambda_{1} c_{1}+\lambda_{3} d\right)-\alpha T_{f}>0
$$

It follows from (19) and (34) that

$$
\mu^{T_{f} / \tau_{a}}\left(\frac{\lambda_{1} c_{1}+\lambda_{3} d}{\lambda_{2}}\right)<e^{-\alpha T_{f}} c_{2} .
$$

By virtue of (33) and (35), we can obtain

$$
x^{T}(t) E^{T} R E x(t)<e^{\alpha t} e^{-\alpha T_{f}} c_{2}=e^{\alpha\left(t-T_{f}\right)} c_{2} ;
$$

then, considering $t<T_{f}$ and $\alpha \geq 0$, we have

$$
x^{T}(t) E^{T} R E x(t)<c_{2} .
$$

Thus, the system (3) is finite-time bounded with respect to $\left(c_{1}, c_{2}, d, R, T_{f}, \sigma\right)$, and the proof is completed.

Remark 10. Since different Lyapunov functions can be constructed for different subsystems, so the multiple Lyapunov functions method is an effective and flexible design tool. Now, the multiple Lyapunov functions have been employed and discussed to study the stability and performance of switched or hybrid systems such as [27-29]. In this paper, the function $V(t)$ in the proof of Theorem 9 is taken as the multiple Lyapunov functions. Compared with the classical Lyapunov function for switched systems of asymptotical stability, there is really no requirement of negative definiteness or negative semidefiniteness on $V(t)$. Actually, if we limit the constants $\alpha<0$ and the exogenous disturbance $w(t)=0$, then $V(t)$ will be a negative definite function. In this case, the system (1) is asymptotically stable on the infinite interval $[0,+\infty)$. We can find the detailed proof in [29].

In order to design controller conveniently, the following conclusion is given to satisfy the condition of Theorem 9. 
Theorem 11. For any $i, j \in\{1,2, \ldots, m\}$, if there exist nonsingular matrix $X_{i}$, matrices $Q_{i}>0, Z_{i}>0$, and scalars $\alpha \geq 0$, $\mu \geq 1$ such that (18) and (19) hold and

$$
\begin{gathered}
E X_{i}=X_{i}^{T} E^{T} \geq 0 \\
{\left[\begin{array}{cc}
A_{i} X_{i}+X_{i}^{T} A_{i}^{T}-\alpha E X_{i} & G_{i} \\
G_{i}^{T} & F_{i}^{T} Q_{i}+Q_{i} F_{i}-\alpha Q_{i}
\end{array}\right]<0} \\
X_{i}^{-T} E=E^{T} R^{1 / 2} Z_{i} R^{1 / 2} E \\
(1-2 \mu) E X_{i}+\mu E X_{j} \leq 0, \quad Q_{i} \leq \alpha Q_{j},
\end{gathered}
$$

then system (3) is regular, pulse-free, and finite-time bounded with respect to $\left(c_{1}, c_{2}, d, R, T_{f}, \sigma\right)$.

Proof. Multiply both sides of (38) separately by $X_{i}^{-T}$ and $X_{i}^{-1}$. Let $X_{i}^{-T}=P$; we can obtain that (14) holds. Similarly, multiply both sides of (39) separately by $\operatorname{diag}\left\{X_{i}^{-T}, I\right\}$ and $\operatorname{diag}\left\{X_{i}^{-1}, I\right\}$. Let $X_{i}^{-T}=P$; then (15) holds. On the other hand, let $X_{i}=P_{i}^{-T}$, it follows from (38) and (41) that

$$
\begin{array}{r}
E P_{i}^{-T}-\alpha E P_{i}^{-T}-\alpha P_{i}^{-1} E^{T}+\alpha E P_{j}^{-T} \leq 0, \\
Q_{i} \leq \alpha Q_{j} .
\end{array}
$$

Now, by virtue of $-\alpha P_{i}^{-1} P_{j} E P_{i}^{-T} \leq-\alpha E P_{i}^{-T}-\alpha P_{i}^{-1} E^{T}+$ $\alpha E P_{j}^{-T}$, it follows from (42) that $E P_{i}^{-T}-\alpha P_{i}^{-1} P_{j} E P_{i}^{-T} \leq 0, Q_{i} \leq$ $\alpha Q_{j}$. Obviously, the previous equation is equivalent to (17), so (41) can ensure that (17) holds. From Theorem 9, it is easy to obtain that system (3) is regular, pulse-free, and finite-time bounded with respect to $\left(c_{1}, c_{2}, d, R, T_{f}, \sigma\right)$.

In the following, we give the following conclusion about the finite-time boundedness problem of system (1) via the action of the state feedback controller $u_{\sigma}(t)=K_{\sigma} x(t)$.

Theorem 12. For any $i, j \in\{1,2, \ldots, m\}$, if there exist nonsingular matrices $X_{i}, L_{i}$, matrices $Q_{i}>0, Z_{i}>0$, and scalars $\alpha \geq 0, \mu \geq 1$ such that (18), (19), and (38)-(41) hold and

$$
\begin{aligned}
& {\left[\begin{array}{cc}
A_{i} X_{i}+X_{i}^{T} A_{i}^{T}+B_{i} L_{i}+L_{i}^{T} B_{i}^{T}-\alpha E X_{i} & G_{i} \\
G_{i}^{T} & F_{i}^{T} Q_{i}+Q_{i} F_{i}-\alpha Q_{i}
\end{array}\right]} \\
& \quad<0,
\end{aligned}
$$

then the system (1) is regular, pulse-free, and finite-time bounded with respect to $\left(c_{1}, c_{2}, d, R, T_{f}, \sigma\right)$ via the action of the state feedback controller $u_{i}(t)=L_{i} X_{i}^{-1} x(t)$.

Proof. According to the proof of Theorem 11, first replace $A_{i}$ with $A_{i}+B_{i} K_{i}$ and let $K_{i} X_{i}=L_{i}$, and then it is easy to obtain the condition of Theorem 12 .

Now, in order to solve by means of the LMI toolbox conveniently, we will process (38) and (40). According to rank $E=r$, there exist invertible matrices $M, N$ such that
$\bar{E}=M E N=\operatorname{diag}\left\{I_{r}, 0\right\}$. Let $\bar{X}_{i}=N^{-1} X_{i} M^{T}$ and from (38) $\bar{X}_{i}$ is given as

$$
\bar{X}_{i}=\left[\begin{array}{cc}
X_{i 1} & 0 \\
X_{i 3} & X_{i 4}
\end{array}\right]
$$

where $X_{i 1}>0, \operatorname{det}\left(X_{i 4}\right) \neq 0$, and $X_{i 3}$ is a matrix with appropriate dimension. In addition, let $\Psi=N\left[\begin{array}{c}0 \\ I_{n-r}\end{array}\right]$ and we can obtain $E \Psi=0$. Based on the above discussion, the following equation holds:

$$
\begin{aligned}
X_{i} & =N\left[\begin{array}{cc}
X_{i 1} & 0 \\
X_{i 3} & X_{i 4}
\end{array}\right] M^{-T} \\
& =N\left(\left[\begin{array}{cc}
X_{i 1} & 0 \\
0 & 0
\end{array}\right]+\left[\begin{array}{cc}
0 & 0 \\
X_{i 3} & X_{i 4}
\end{array}\right]\right) M^{-T} \\
& =N \Omega_{i} N^{T} E^{T}+\Psi Y_{i} M^{-T},
\end{aligned}
$$

where $\Omega_{i}=\operatorname{diag}\left\{X_{i 1}, \#\right\}>0$ and $Y_{i}=\left[\begin{array}{ll}X_{i 3} & X_{i 4}\end{array}\right]$. It is obvious that $X_{i}=N \Omega_{i} N^{T} E^{T}+\Psi Y_{i} M^{-T}$ satisfies (41) and (43). Let $Z_{i}=R^{-1 / 2} M^{T} \Omega_{i}^{-1} M R^{-1 / 2}$, and then one obtains that (40) holds.

Equation (18) can be guaranteed by the following LMIs. For any $i, j \in\{1,2, \ldots, m\}$, there exist scalars $\varepsilon_{1}, \varepsilon_{2}$ such that

$$
\begin{gathered}
\varepsilon_{1} I<R^{1 / 2} M^{-1} \Omega_{i} M^{-T} R^{1 / 2}<I, \\
Q_{i}<\varepsilon_{2} I, \\
{\left[\begin{array}{cc}
-e^{\alpha T_{f}} c_{2}+\varepsilon_{2} d & -\sqrt{c_{1}} \\
-\sqrt{c_{1}} & -\varepsilon_{1}
\end{array}\right]<0 .}
\end{gathered}
$$

Theorem 13. For any $i, j \in\{1,2, \ldots, m\}$, if there exist matrices $\Omega_{i}=\operatorname{diag}\left\{X_{i 1}, \#\right\}>0, Q_{i}>0, L_{i}, Y_{i}$ and scalars $\alpha \geq 0, \mu \geq 1$ such that (19) and (46) hold and

$$
\begin{gathered}
(1-2 \mu) E N \Omega_{i} N^{T} E^{T}+\mu E N \Omega_{j} N^{T} E^{T} \leq 0, \\
Q_{i} \leq \alpha Q_{j}, \\
{\left[\begin{array}{cc}
\Xi_{i} & G_{i} \\
G_{i}^{T} & F_{i}^{T} Q_{i}+Q_{i} F_{i}-\alpha Q_{i}
\end{array}\right]<0,}
\end{gathered}
$$

then the switched descriptor system (1) is regular, pulse-free, and finite-time bounded with respect to $\left(c_{1}, c_{2}, d, R, T_{f}, \sigma\right)$ via the action of the state feedback controller $u_{i}(t)=L_{i} \beta^{-1}\left(\Omega_{i}\right.$, $\left.Y_{i}\right) x(t)$, where

$$
\begin{aligned}
\Xi_{i}= & A_{i} \beta\left(\Omega_{i}, Y_{i}\right)+\beta^{T}\left(\Omega_{i}, Y_{i}\right) A_{i}^{T} \\
& +B_{i} L_{i}+L_{i}^{T} B_{i}^{T}-\alpha E \beta\left(\Omega_{i}, Y_{i}\right),
\end{aligned}
$$

and the matrix $\beta\left(\Omega_{i}, Y_{i}\right)=N \Omega_{i} N^{T} E^{T}+\Psi Y_{i} M^{-T}$ is nonsingular; matrices $M, N$ satisfy $M E N=\operatorname{diag}\left\{I_{r}, 0\right\}, \Psi=$ $N\left[\begin{array}{ll}0 & I_{n-r}\end{array}\right]^{T}$ 
Remark 14. If there exists $E=I, F_{i}=0$, we can obtain the sufficient condition of finite-time boundedness for switched systems with constant disturbance based on the above conclusion. If there exists $w(t)=0$, and $V(t)$ is taken as common Lyapunov function, then the system is of consistent finite-time boundedness with arbitrary switching signals.

Theorem 15. Consider the switched descriptor system as follows:

$$
E \dot{x}(t)=A_{\sigma(t)} x(t)+B_{\sigma(t)} u_{\sigma(t)}(t) .
$$

For any $i, j \in\{1,2, \ldots, m\}$, if there exist matrices $\Omega_{i}=$ $\operatorname{diag}\left\{X_{i 1}, \#\right\}>0, L_{i}, Y_{i}$ and scalars $\alpha \geq 0, \mu \geq 1$ such that

$$
\begin{gathered}
(1-2 \mu) E N \Omega_{i} N^{T} E^{T}+\mu E N \Omega_{j} N^{T} E^{T} \leq 0, \\
A_{i} \beta\left(\Omega_{i}, Y_{i}\right)+\beta^{T}\left(\Omega_{i}, Y_{i}\right) A_{i}^{T} \\
+B_{i} L_{i}+L_{i}^{T} B_{i}^{T}-\alpha E \beta\left(\Omega_{i}, Y_{i}\right)<0, \\
\varepsilon_{1} I<R^{1 / 2} M^{-1} \Omega_{i} M^{-T} R^{1 / 2}<\varepsilon_{2} I, \\
\varepsilon_{2} c_{1}<\varepsilon_{1} c_{2}
\end{gathered}
$$

and the average dwell time satisfies

$$
\tau_{a}>\tau_{a}^{*}=\frac{T_{f} \ln \mu}{\ln \left(\lambda_{2} c_{2}\right)-\ln \left(\lambda_{1} c_{1}\right)-\alpha T_{f}},
$$

then the switched descriptor system (49) is regular, pulse-free, and finite-time stable with respect to $\left(c_{1}, c_{2}, d, R, T_{f}, \sigma\right)$ via the action of the state feedback controller $u_{i}(t)=L_{i} \beta^{-1}\left(\Omega_{i}, Y_{i}\right) x(t)$, where $\beta\left(\Omega_{i}, Y_{i}\right)=N \Omega_{i} N^{T} E^{T}+\Psi Y_{i} M^{-T}$ is nonsingular and matrices $M, N$ satisfy $M E N=\operatorname{diag}\left\{I_{r}, 0\right\}, \Psi=N\left[\begin{array}{ll}0 & I_{n-r}\end{array}\right]^{T}$.

\section{Numerical Simulations}

Example 16. Consider the continuous switched descriptor system (1) with parameters as follows:

$$
\begin{aligned}
& A_{1}=\left[\begin{array}{ccc}
-2.1 & 1.0 & -0.3 \\
-0.8 & -0.4 & 0.4 \\
0.8 & -0.6 & 1.0
\end{array}\right] \\
& B_{1}=\left[\begin{array}{c}
1 \\
-1 \\
1
\end{array}\right], \quad G_{1}=\left[\begin{array}{cc}
0.2 & 0.1 \\
-0.1 & 0.3 \\
0.1 & -0.1
\end{array}\right] \text {, } \\
& F_{1}=\left[\begin{array}{cc}
0 & 0.1 \\
-0.1 & 0
\end{array}\right] \text {, } \\
& A_{2}=\left[\begin{array}{ccc}
-2.6 & -0.5 & 0.5 \\
0.6 & 0.5 & -0.8 \\
-0.5 & 0.2 & 1.0
\end{array}\right] \text {, } \\
& B_{2}=\left[\begin{array}{c}
-1 \\
1 \\
1
\end{array}\right], \quad G_{2}=\left[\begin{array}{cc}
-0.1 & 0.2 \\
0.2 & -0.1 \\
-0.1 & 0.3
\end{array}\right] \text {, } \\
& F_{2}=\left[\begin{array}{cc}
0 & 0.1 \\
-0.1 & 0
\end{array}\right]
\end{aligned}
$$

The values of $E, \alpha, \mu, c_{1}, c_{2}, d, T_{f}$, and $R$ are given as follows: $\alpha=0.3, \mu=1.2, E=\operatorname{diag}\{1,1,0\}$, let $c_{1}=2, c_{2}=10, d=3$, $T_{f}=1$, and $R=I$.

Then, according to Theorem 13, we get

$$
\begin{gathered}
\Omega_{1}=\left[\begin{array}{ccc}
0.5249 & 0.0266 & 0 \\
0.0266 & 0.4635 & 0 \\
0 & 0 & 0.4749
\end{array}\right], \\
\Omega_{2}=\left[\begin{array}{ccc}
0.5353 & 0.0275 & 0 \\
0.0275 & 0.4787 & 0 \\
0 & 0 & 0.4749
\end{array}\right], \\
Y_{1}=\left[\begin{array}{lll}
-0.5388 & -0.2418 & -0.5585
\end{array}\right], \\
Y_{2}=\left[\begin{array}{lll}
1.2591 & -0.2093 & -1.1868
\end{array}\right], \\
L_{1}=\left[\begin{array}{lll}
0.2230 & 0.4453 & -0.2745
\end{array}\right], \\
L_{2}=[-0.0427-1.17100 .3543], \\
Q_{1}=\left[\begin{array}{cc}
2.1001 & 0 \\
0 & 2.1001
\end{array}\right], \\
Q_{2}=\left[\begin{array}{cc}
2.1001 & 0 \\
0 & 2.1001
\end{array}\right] .
\end{gathered}
$$

In the following, we have by calculating

$$
\begin{aligned}
& \beta\left(\Omega_{1}, Y_{1}\right)=\left[\begin{array}{ccc}
0.5453 & 0.0266 & 0 \\
0.0266 & 0.4635 & 0 \\
-0.5388 & -0.2418 & -0.5585
\end{array}\right], \\
& \beta\left(\Omega_{2}, Y_{2}\right)=\left[\begin{array}{ccc}
0.5353 & 0.0275 & 0 \\
0.0275 & 0.4787 & 0 \\
1.2591 & -0.2093 & -1.1868
\end{array}\right] .
\end{aligned}
$$

Then we can obtain the designed state feedback controllers as follows:

$$
\begin{aligned}
u_{1}(t) & =L_{1} \beta^{-1}\left(\Omega_{1}, Y_{1}\right) x(t) \\
& =\left[\begin{array}{lll}
0.8376 & 1.1691 & 0.4914
\end{array}\right] x(t), \\
u_{2}(t) & =L_{2} \beta^{-1}\left(\Omega_{2}, Y_{2}\right) x(t) \\
& =\left[\begin{array}{lll}
0.7566 & -2.6204 & -0.2985
\end{array}\right] x(t) .
\end{aligned}
$$

Furthermore, we can obtain $\lambda_{1}=2.1949, \lambda_{2}=1.8078, \lambda_{3}=$ 2.1001, and $\tau_{a}>\tau^{*}=0.2517$. For any switching signal $\sigma(t)$ with average dwell time $\tau_{a}>0.2517$, the switched descriptor system (1) is finite-time stable.

When $w(t)=\left[\begin{array}{l}\sin (0.1 t) \\ \cos (0.1 t)\end{array}\right]$, the trajectory of $x^{T}(t) E^{T} R E x(t)$ is presented in Figure 1 and the switching signal is shown in Figure 2. 


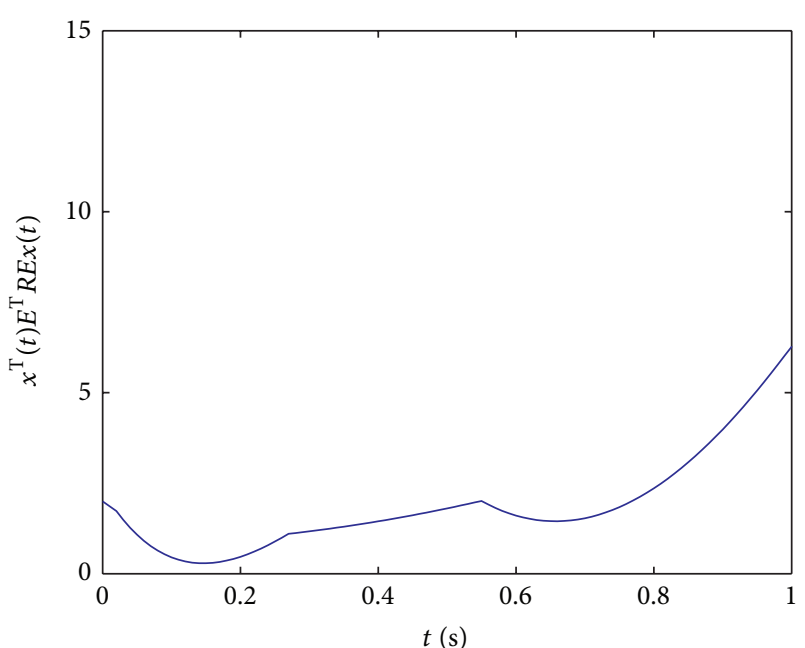

Figure 1: Trajectory of $x^{T}(t) E^{T} R E x(t)$.

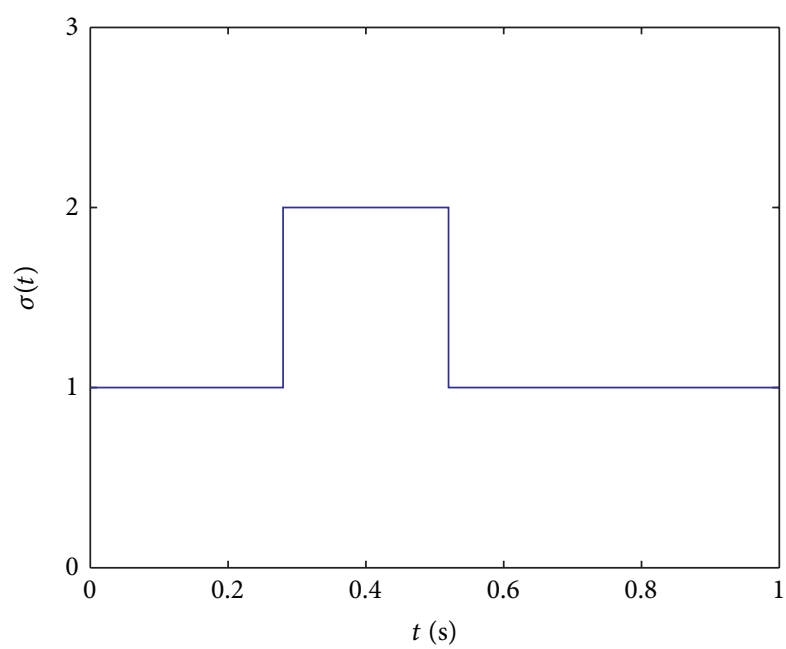

Figure 2: Switched signal $\sigma(t)$.

Example 17. Consider the continuous switched descriptor system (49) with parameters as follows:

$$
\begin{array}{ll}
A_{1}=\left[\begin{array}{ccc}
-1 & -2 & 1 \\
2 & -1 & 3 \\
0 & 1 & -2
\end{array}\right], & B_{1}=\left[\begin{array}{c}
-1 \\
-9 \\
1
\end{array}\right], \\
A_{2}=\left[\begin{array}{ccc}
-2 & 1 & -1 \\
1 & -1 & 0 \\
2 & -2 & -1
\end{array}\right], & B_{1}=\left[\begin{array}{c}
3 \\
-8 \\
-1
\end{array}\right] .
\end{array}
$$

Choosing the matrix $E=\operatorname{diag}\{1,1,0\}$ and letting $\alpha=0.1, \mu=$ $1.2, c_{1}=1, c_{2}=5, T_{f}=6$, and $R=I$, by virtue of Theorem 15 , the feasible solutions are given as

$$
\begin{gathered}
\Omega_{1}=\left[\begin{array}{ccc}
33.4670 & -2.1549 & 0 \\
-2.1549 & 37.2884 & 0 \\
0 & 0 & 36.9815
\end{array}\right], \\
\Omega_{2}=\left[\begin{array}{ccc}
31.4690 & 0.5435 & 0 \\
0.5435 & 38.9031 & 0 \\
0 & 0 & 36.9815
\end{array}\right], \\
Y_{1}=\left[\begin{array}{lll}
6.8444 & 21.3449 & 15.4656
\end{array}\right] \\
Y_{2}=\left[\begin{array}{lll}
-1.3833 & -31.4843 & 32.2021
\end{array}\right], \\
L_{1}=\left[\begin{array}{lll}
3.7061 & 5.1168 & 5.1099
\end{array}\right], \\
L_{2}=\left[\begin{array}{lll}
11.9850 & -1.8186 & -5.5726
\end{array}\right]
\end{gathered}
$$

Now, it can be obtained as

$$
\begin{aligned}
& \beta\left(\Omega_{1}, Y_{1}\right)=\left[\begin{array}{ccc}
33.4670 & -2.1549 & 0 \\
-2.1549 & 37.2844 & 0 \\
6.8444 & 21.3449 & 15.4656
\end{array}\right], \\
& \beta\left(\Omega_{2}, Y_{2}\right)=\left[\begin{array}{ccc}
31.4690 & 0.5435 & 0 \\
0.5435 & 38.9031 & 0 \\
-1.3833 & -31.4843 & 32.2021
\end{array}\right] .
\end{aligned}
$$

Then we can obtain the designed controllers as follows:

$$
\begin{aligned}
u_{1}(t) & =L_{1} \beta^{-1}\left(\Omega_{1}, Y_{1}\right) x(t) \\
& =\left[\begin{array}{lll}
0.0400 & -0.0496 & 0.3304
\end{array}\right] x(t), \\
u_{2}(t) & =L_{2} \beta^{-1}\left(\Omega_{2}, Y_{2}\right) x(t) \\
& =\left[\begin{array}{lll}
0.3766 & -0.1921 & -0.1731
\end{array}\right] x(t) .
\end{aligned}
$$

By calculating, we have $\lambda_{1}=0.0318, \lambda_{2}=0.0257$, and $\tau_{a}>$ $\tau^{*}=1.3758$. For any switching signal $\sigma(t)$ with average dwell time $\tau_{a}>1.3758$, the system (49) is finite-time stable with respect to $\left(c_{1}, c_{2}, d, R, T_{f}, \sigma\right)$ via the action of the state feedback controller. Figure 3 shows the trajectory of $x^{T}(t) E^{T} R E x(t)$, and Figure 4 shows the switching signal.

If the switching is too frequent, it is possible that the whole system is not finite-time stable. For instance, given the switching signal as follows:

$$
\sigma(t)= \begin{cases}1, & x_{2} x_{3}-x_{1} x_{2}<0 \\ 2, & x_{2} x_{3}-x_{1} x_{2} \geq 0\end{cases}
$$

the whole system is not finite-time stable any more. The trajectory of $x^{T}(t) E^{T} R E x(t)$ is shown in Figure 5 and the switching signal $\sigma(t)$ is shown in Figure 6.

\section{Conclusion}

In this paper, the issues of finite-time stability and finite-time boundedness for a class of continuous switched descriptor systems have been studied. The sufficient and necessary condition of finite-time stability for switched descriptor systems is presented by applying the state transition matrix method. 


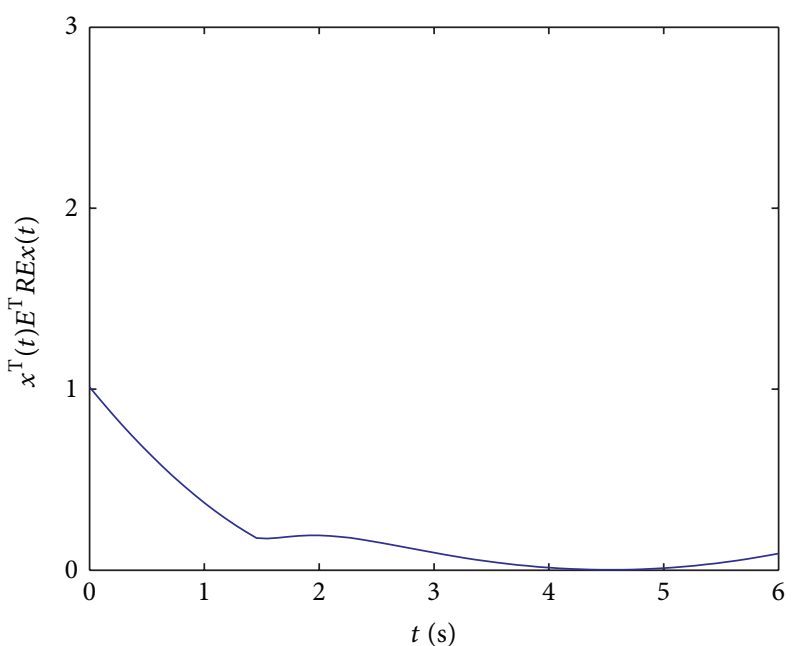

Figure 3: Trajectory of $x^{T}(t) E^{T} R E x(t)$.

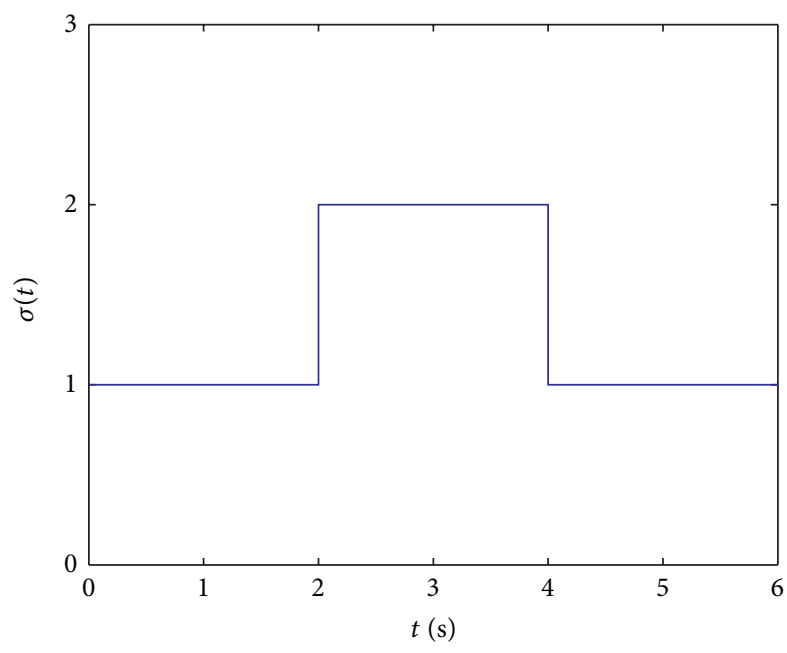

FIGURE 4: Switched signal $\sigma(t)$.

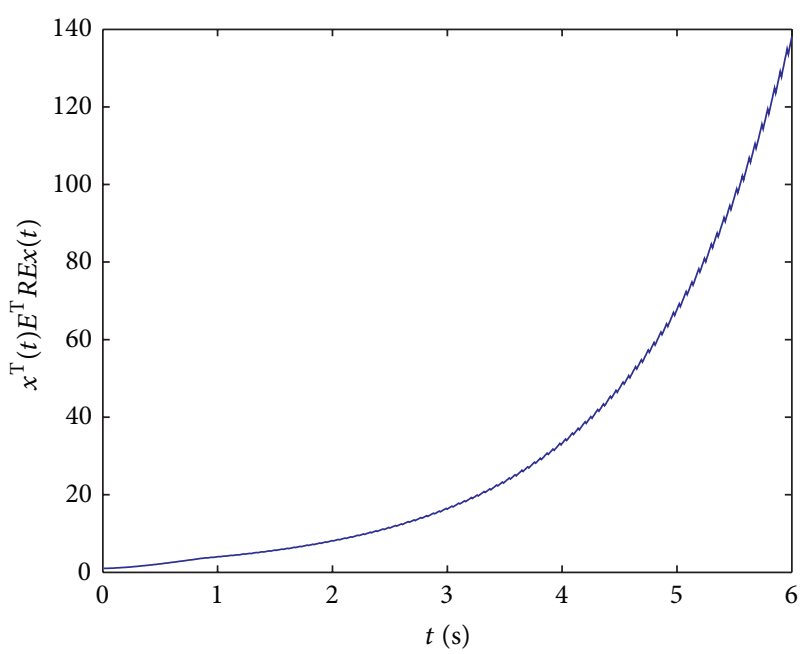

Figure 5: Trajectory of $x^{T}(t) E^{T} R E x(t)$.

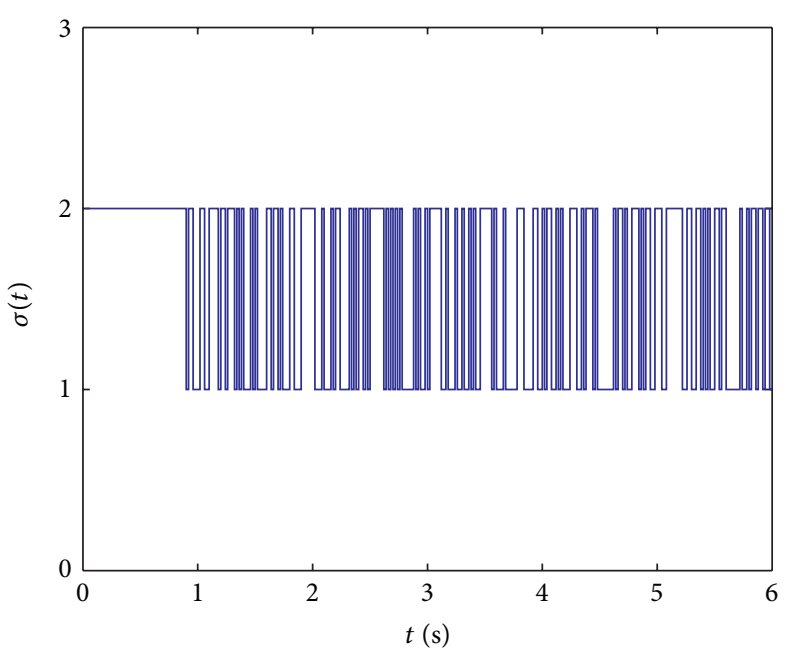

FIGURE 6: Switching signal $\sigma(t)$.

The obtained condition has certain theoretical value, but it also has two disadvantages in practical application. First, it is difficult to calculate the state transition matrix; on the other hand, it is inconvenient to design controller. In order to solve these problems, based on the average dwell time approach and the multiple Lyapunov function technique, the existence of state feedback controllers is proposed with arbitrary switching rules, which guarantee that the switched descriptor systems are finite-time stable and finite-time bounded, respectively. More possible future works are to consider output feedback stabilization for the uncertain switched descriptor systems with time-varying delay.

\section{Conflict of Interests}

The authors declare that there is no conflict of interests regarding the publication of this paper.

\section{Acknowledgments}

This study was supported by the National Natural Science Foundation of China (Grant no. 61174032), the Natural Science Foundation of Jiangsu Province (Grant no. BK2012550), and the Fundamental Research Funds for the Central University (Grant no. JUDCF11040).

\section{References}

[1] S. Shi, Q. Zhang, Z. Yuan, and W. Liu, "Hybrid impulsive control for switched singular systems," IET Control Theory \& Applications, vol. 5, no. 1, pp. 103-111, 2011.

[2] G. Xie and L. Wang, "Stability and stabilization of switched descriptor systems under arbitrary switching," in Proceedings of the IEEE International Conference on Systems, Man and Cybernetics (SMC'04), pp. 779-783, Hague, The Netherlands, October 2004.

[3] Y. J. Yin and J. Zhao, "Stability of switched linear singular systems with impulsive effects," Acta Automatica Sinica, vol. 33, no. 4, pp. 446-448, 2007. 
[4] T. C. Wang and Z. R. Gao, "Asymptotic stability criterion for a class of switched uncertain descriptor systems with time-delay," Acta Automatica Sinica, vol. 34, no. 8, pp. 1013-1016, 2008.

[5] J.-X. Lin and S.-M. Fei, "Robust exponential admissibility of uncertain switched singular time-delay systems," Acta Automatica Sinica, vol. 36, no. 12, pp. 1773-1779, 2010.

[6] S. P. Ma, C. H. Zhang, and Z. Wu, "Delay-dependent stability of $H_{\infty}$ control for uncertain discrete switched singular systems with time-delay," Applied Mathematics and Computation, vol. 206, no. 1, pp. 413-424, 2008.

[7] H. J. Gao and Y. Q. Wu, "A design scheme of variable structure $H$-infinity control for uncertain singular Markov switched systems based on linear matrix inequality method," Nonlinear Analysis: Hybrid Systems, vol. 1, no. 3, pp. 306-316, 2007.

[8] D. Koenig and B. Marx, " $H_{\infty}$-filtering and state feedback control for discrete-time switched descriptor systems," IET Control Theory \& Applications, vol. 3, no. 6, pp. 661-670, 2009.

[9] Z.-R. Gao and Z.-C. Ji, "Optimal guaranteed cost control for a class of switched singular systems with time-delay," Systems Engineering and Electronics, vol. 33, no. 6, pp. 1358-1361, 2011.

[10] Z.-Q. Gu, H.-P. Liu, F.-C. Liao, and Y.-J. Wang, "Guaranteed cost control for uncertain time-delay switched singular systems based on LMI," Systems Engineering and Electronics, vol. 32, no. 1, pp. 147-151, 2010.

[11] K. Yang, Y. X. Shen, and Z. C. Ji, "Robust $H_{\infty}$ Guaranteed cost control for uncertain switched descriptor delayed systems with nonlinear disturbance," Proceedings of the IMechE-part I: Journal of Systems and Control Engineering, vol. 227, no. 9, pp. 686-691, 2013.

[12] B. Meng and J.-F. Zhang, "Reachability conditions for switched linear singular systems," IEEE Transactions on Automatic Control, vol. 51, no. 3, pp. 482-488, 2006.

[13] B. Meng and J. Zhang, "Reachability analysis of switched linear discrete singular systems," Journal of Control Theory and Applications, vol. 4, no. 1, pp. 11-17, 2006.

[14] P. Dorato, "Short time stability in linear time-varying systems," in Proceedings of the IRE International Convention Record, pp. 83-87, New York, NY, USA, 1961.

[15] X. Z. Lin, H. B. Du, and S. H. Li, "Finite-time boundedness and $L_{2}$-gain analysis for switched delay systems with normbounded disturbance," Applied Mathematics and Computation, vol. 217, no. 12, pp. 5982-5993, 2011.

[16] G. L. Zhao and J. C. Wang, "Finite time stability and $L_{2}$ gain analysis for switched linear systems with state-dependent switching," Journal of the Franklin Institute, vol. 350, no. 5, pp. 1075-1092, 2013.

[17] X. Lin, H. Du, S. Li, and Y. Zou, "Finite-time stability and finitetime weighted $L_{2}$-gain analysis for switched systems with timevarying delay," IET Control Theory \& Applications, vol. 7, no. 7, pp. 1058-1069, 2013.

[18] H. Liu, Y. Shen, and X. Zhao, "Finite-time stabilization and boundedness of switched linear system under state-dependent switching," Journal of the Franklin Institute, vol. 350, no. 3, pp. 541-555, 2013.

[19] X. Lin, H. Du, S. Li, and Y. Zou, "Finite-time boundedness and finite-time $l_{2}$ gain analysis of discrete-time switched linear systems with average dwell time," Journal of the Franklin Institute, vol. 350, no. 4, pp. 911-928, 2013.

[20] Z. Xiang, C. Qiao, and M. S. Mahmoud, "Finite-time analysis and $H_{\infty}$ control for switched stochastic systems," Journal of the Franklin Institute, vol. 349, no. 3, pp. 915-927, 2012.
[21] J.-E. Feng, Z. Wu, and J.-B. Sun, "Finite-time control of linear singular systems with parametric uncertainties and disturbances," Acta Automatica Sinica, vol. 31, no. 4, pp. 634-637, 2005.

[22] S. Zhao, J. Sun, and L. Liu, "Finite-time stability of linear timevarying singular systems with impulsive effects," International Journal of Control, vol. 81, no. 11, pp. 1824-1829, 2008.

[23] Y. Zhang, C. Liu, and X. Mu, "Robust finite-time $H_{\infty}$ control of singular stochastic systems via static output feedback," Applied Mathematics and Computation, vol. 218, no. 9, pp. 5629-5640, 2012.

[24] Z. Su, Q. L. Zhang, and J. Ai, "Practical and finite-time fuzzy adaptive control for nonlinear descriptor systems with uncertainties of unknown bound," International Journal of Systems Science, vol. 44, no. 12, pp. 2223-2233, 2013.

[25] Z. R. Gao, Y. X. Shen, and Z. C. Ji, "Uniform finite-time stability of discrete-time switched descriptor systems," Acta Physica Sinica, vol. 61, no. 12, Article ID 120203, 2012.

[26] K. Yang, Y. X. Shen, and Z. C. Ji, "Uniform finite-time stability for a class of continuous-time switched descriptor systems with time-varying delay," Control and Decision. In press.

[27] L. Long and J. Zhao, " $H_{\infty}$ control of switched nonlinear systems in $p$-normal form using multiple Lyapunov functions," IEEE Transactions on Automatic Control, vol. 57, no. 5, pp. 1285-1291, 2012.

[28] M. A. Müller and D. Liberzon, "Input/output-to-state stability and state-norm estimators for switched nonlinear systems," Automatica, vol. 48, no. 9, pp. 2029-2039, 2012.

[29] X.-M. Sun, J. Zhao, and D. J. Hill, "Stability and $L_{2}$-gain analysis for switched delay systems: a delay-dependent method," Automatica, vol. 42, no. 10, pp. 1769-1774, 2006. 


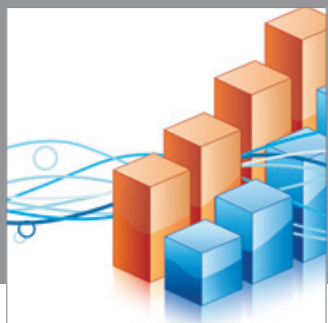

Advances in

Operations Research

mansans

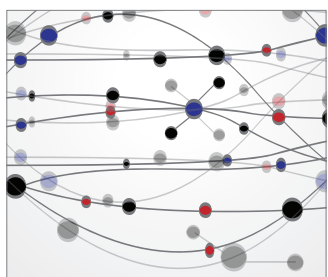

The Scientific World Journal
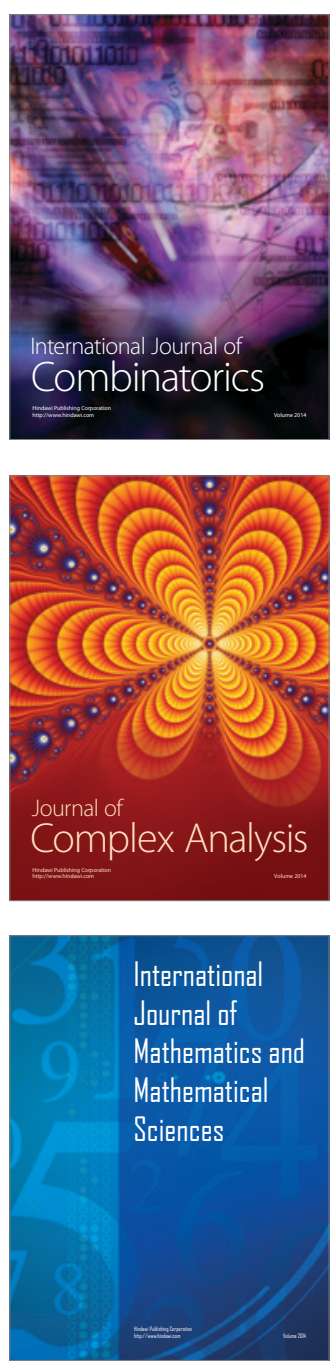
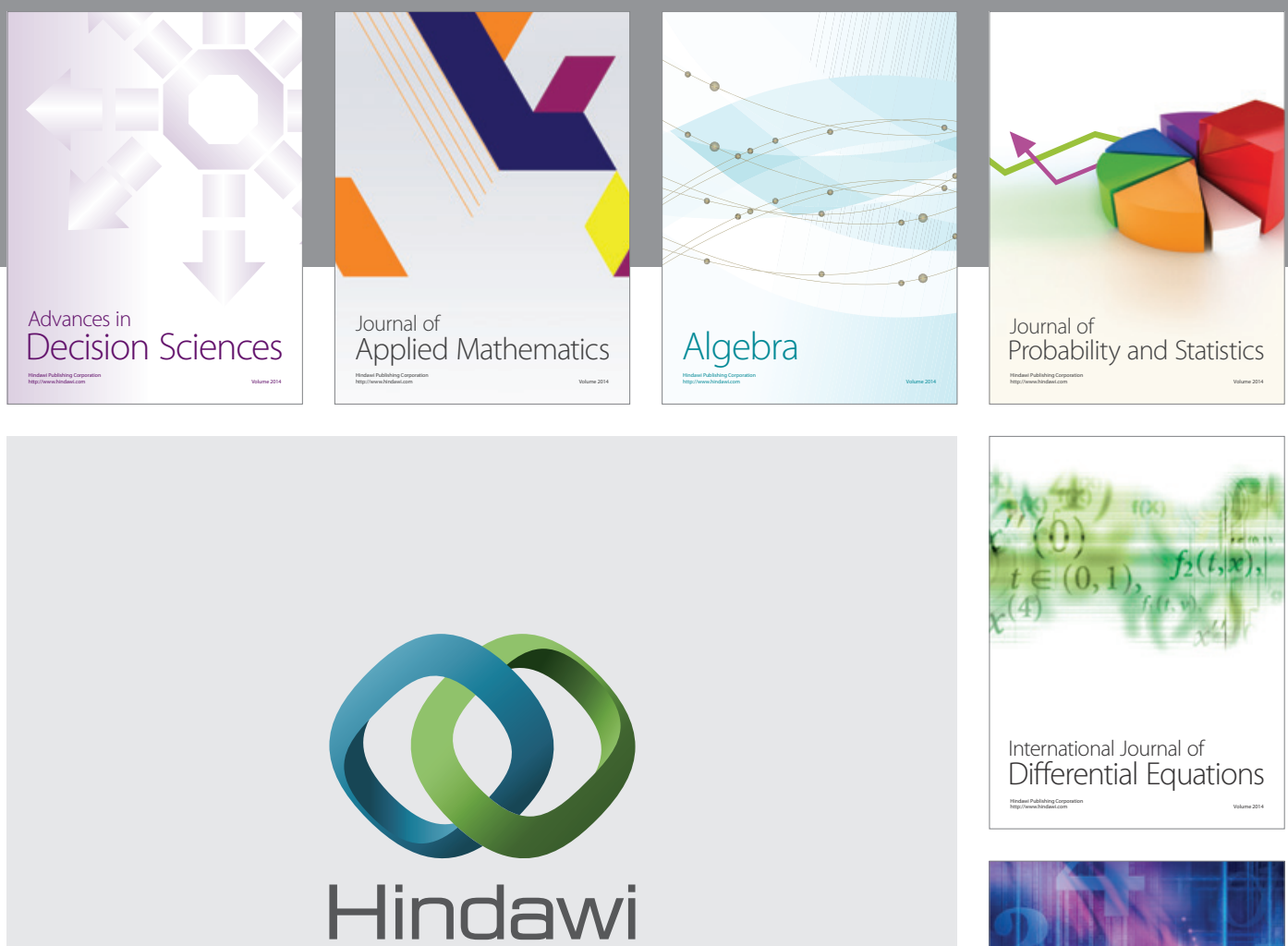

Submit your manuscripts at http://www.hindawi.com
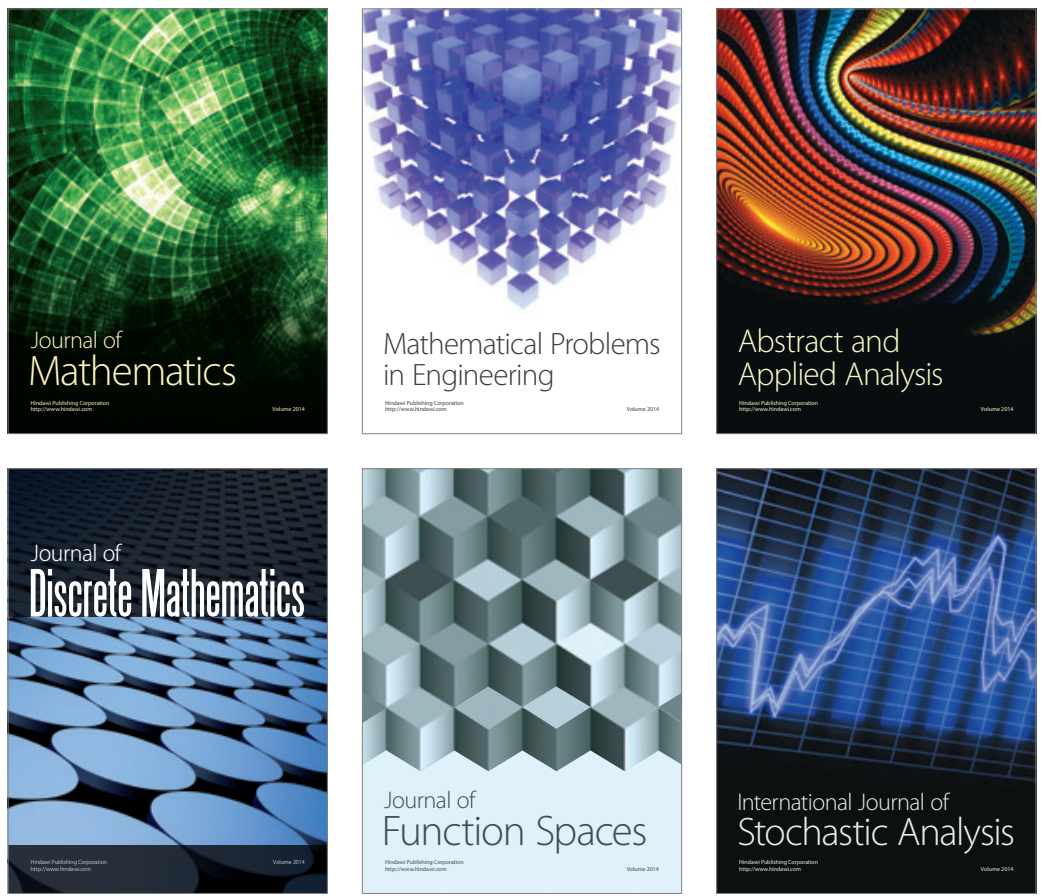

Journal of

Function Spaces

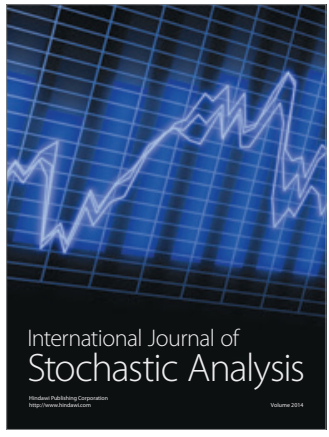

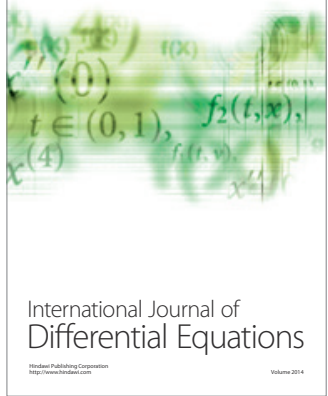
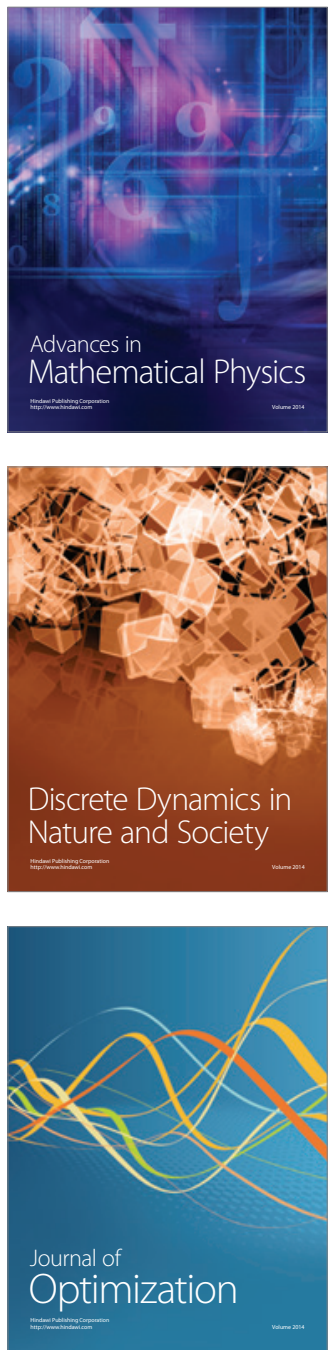